\title{
A Novel Algorithm for Face Recognition From Very Low Resolution Images
}

\author{
C. Senthilsingh ${ }^{\dagger}$ and M. Manikandan*
}

\begin{abstract}
Face Recognition assumes much significance in the context of security based application. Normally, high resolution images offer more details about the image and recognizing a face from a reasonably high resolution image would be easier when compared to recognizing images from very low resolution images. This paper addresses the problem of recognizing faces from a very low resolution image whose size is as low as $8 \times 8$. With the use of CCTV(Closed Circuit Television) and with other surveillance camera-based application for security purposes, the need to overcome the shortcomings with very low resolution images has been on the rise. The present day face recognition algorithms could not provide adequate performance when employed to recognize images from VLR images. Existing methods use super-resolution (SR) methods and Relation Based Super Resolution methods to construct from very low resolution images. This paper uses a learning based super resolution method to extract and construct images from very low resolution images. Experimental results show that the proposed SR algorithm based on relationship learning outperforms the existing algorithms in public face databases.
\end{abstract}

Keywords: Face recognition, Face super-resolution (SR), Relationship learning, Very low resolution (VLR).

\section{Introduction}

The face recognition research is not in its infancy and it has been more than 36 years since researchers have started solving the problem of recognizing faces. Day by day, new application that involves face recognition are being deployed. As the research has been pursued on this field for a very long time, the researchers have started believing that saturation has reached in this field. Immediately it is realized that with security based application like surveillance camera and CCTV, people realized that the need to find the solution has gathered momentum. In order to maximize camera viewing and covering an area, wide area cameras are used. The relative area covered by the face image of the person is very small compared to the total area of the image and in most of the cases, especially when the person is at long distance, the pixel resolution of the image would be even less that $8 \times 8$. Working on such a very low resolution (VLR) face image is called a VLR face problem.

Minimum size required to reconstruct an image from a low resolution is found out to be between $32 \times 32$ and $64 \times$ 64 [17]. It further states that any image which is found to be less than $32 \times 32$ will have less detail, such as the recognition performance be reduced [9]. Fig. 1(a), (b) gives a complete scenario of the stated problem.

Fig. 1 (b) clearly elucidates the amount of details lost

$\dagger$ Corresponding Author: Dept. of Electronics, Anna University, Chennai. (senthilsingh @gmail.com)

* Dept. of Electronics, Anna University, Chennai. (maniiz@gmail.com) Received: July 13, 2014; Accepted: October 1, 2014 when the face region of the image is zoomed. This VLR problem can be solved if we could supply the missing details in the image, Therefore, super-resolution (SR) algorithms has been employed. [3, 4, 6, 7]. Super resolution employs two methods of reconstructing the image from the VLR data base. The reconstruction of images based on super resolution, because of the self-similarity of the face images, learning-based SR algorithms are always used to enhance the resolution of the face images.

In the literature, different kinds of super resolution algorithms [1, 5, 8-10, 15, 16, 18, 19] have been developed. Many of the stated algorithms are based on two approaches: maximum a posteriori (MAP) and examplebased approaches. Error for the reconstructed image is measured using data constraint [15].

In a surveillance system, the resolution of face image is normally low in the video. Hence the details of facial

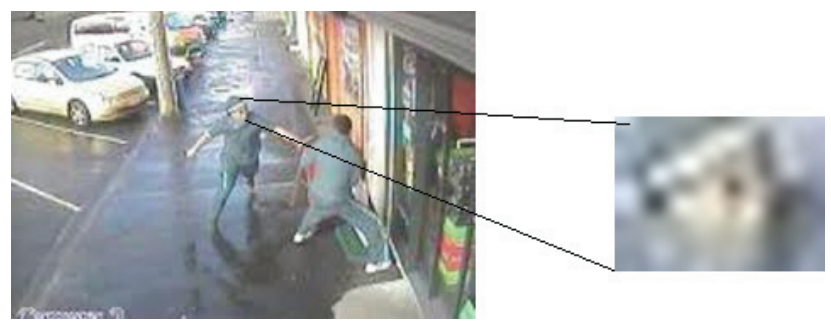

Fig. 1. Courtesy: www.3news.co.nz Typical frame from a surveillance-CCTV camera. The persons seen are stealing from the shop: (a) Surveillance video; (b) Face region. 
features which are available in the respective high resolution image are very important to compare and identify the face from the low resolution images. Hence, a LR image is obtained from HR images by smoothing and down-sampling. It is expected that it must be close to the input LR image. Therefore, this constraint called data constraint is introduced. Here, the images are compared by calculating the distance in the low-resolution (LR) image space for SR processing. For example, Baker and Kanade's method [1] copies the high-resolution (HR) details from HR training images, which are the best match in the corresponding LR training images, as the missing details in the input query LR image.

There are other constraints to be looked after to get a good match in the case of very low resolution images. These constraints are stated clearly in [15].

(a) Global constraint. The result must have common characteristics of a human face, e.g. eyes, mouth and nose, symmetry, etc. The facial features should be coherent.

(b) Local constraint. The result must have specific characteristics of this face image, with photorealistic local features. The global face similarity constraint and local constraints are important to avoid noise and to have match with ordinary facial features.

For this method to be successful, the distance metric calculated in the VLR image space must reflect the actual face similarity in the HR image space. However, the studies based on empirical results [17] show that the face similarity measure in the VLR image space cannot reflect the actual face similarity of HR face images. As such, many SR algorithms in the current literature may not be successful to solve the VLR problem.

Later, VLR problem was given a new approach namely relationship-learning-based SR (RLSR) approach [20]. The RLSR algorithm has significantly reduced the effect of the VLR problem. RLSR algorithm is a type of algorithm which defines a mapping (called relationship) from the VLR image space to the HR image space for HR image reconstruction. Humans can identify the images and machines may also need to identify the images. Each requires different approaches. For humans to identify the image, the image quality needs to be good. In order to achieve that, RLSR algorithm has introduced a new data constraint and in case of machine based recognition, a discriminative constraint is used as to enable the machines to identify the images. It is based on discriminative features.

In Fig. 2(a) the circles represent images. Existing algorithms evaluate the error in the LR image space by an old data constraint; the proposed method makes use of a new data constraint that does measurement in the HR image space. In Fig. 2(b) clearly gives an overview of the RLSR algorithm. This algorithm makes use of the information that may be available during the training phase.

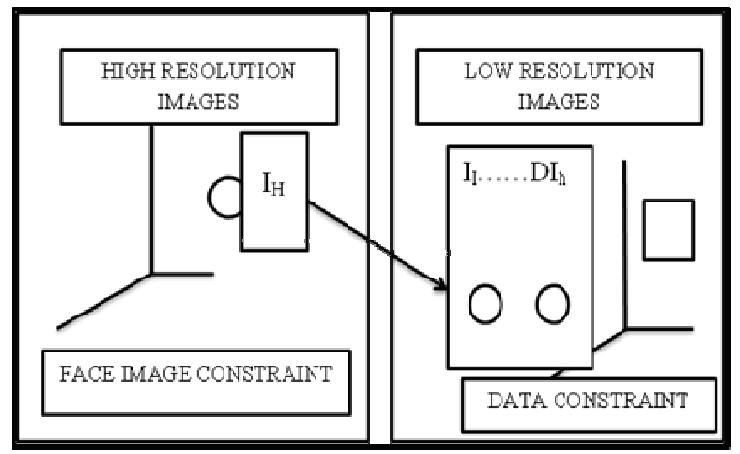

Fig. 2. (a) Existing methods.

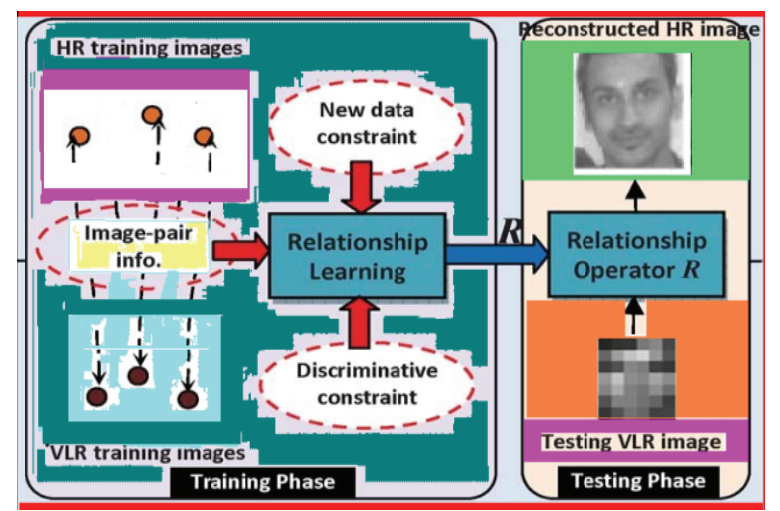

Fig.2. (b) Proposed method

SR and LSR methods normally recover the images directly from the VLR image(s) and the training images. But, the RLSR algorithm does the following:

First, a relationship VLR and HR image spaces is established. Secondly, the outcome is applied to recover the high resolution images from the low resolution images.

In this paper, a few changes have been incorporated to improve the performance of the RLSR algorithm and better results are obtained.

In order to establish the relationship in HR image space, regression analysis is done. The error measurement in HR image space is found using the new data constraint and is introduced with the help of regression model. Ordinary least squares is used. This method minimizes the sum of squared vertical distances between the observed responses in the dataset and responses predicted by the linear approximation. As such the proposed method recovers images with more details and handles the very low resolution problem better.

$\mathrm{K}$-means clustering algorithm is proposed to avoid the over fitting effect of the Ordinary least squares method. This new clustering algorithm maximizes the linearity of the data in each cluster. This clustering ensures data linearity in each cluster. Therefore, the linearity-clusteringbased relationship learning method can handle the complex nonlinear case.

In order to categorize and to enhance the differentiability of the face image, a categorizing constraint is integrated 
in the optimization function with the help of class label information

\section{Related Works}

Many super resolution methods have been introduced in the literature SR $[1,9-13,15,16,18-20]$ to meet the growing demand and challenges of the face recognition applications. Almost all the stated SR algorithms are machine learning based. The machine based approach basically does the following: Given a set of training LRHR image pairs $\left(I_{l}^{i}, \mathrm{I}_{h}^{i}\right)$ and an input query LR image, the SR algorithm reconstructs an HR image from and training data.

These algorithms normally follows two approaches, namely, MAP and example-based. The reconstructed image in both the method is verified with the help of data constraint Both approaches employ the same data constraint to verify the reconstructed HR images. But the inherent shortcomings that exist in the data constrain creates many problems and hence, it may not work with the VLR problem. In order to overcome this difficulty, the association relation based approach learns a relationship between the input VLR image space and HR images which is explained in the Fig. 2(b). Unlike most of the existing methods recovering the images directly from the VLR image(s) and the training images, our method first determines relationship between VLR and HR image spaces and then applies on VLR images to recover the HR ones. Very low resolution face recognition problem by W. W. Zou and P. C. Yuen [20], formed where the VLR problem was introduced and a new ALSR algorithm was proposed to alleviate it. Super-resolution from a single image [7] proposes a unified framework for combining the two general approaches of super resolution. It show how this combined approach can be applied to obtain super resolution from as little as a single image (with no database).

Learning the relationship between high and low resolution images in kernel space for face super resolution [20] proposes a new nonlinear face super resolution algorithm to address recognition of low resolution face image with nonlinear variations. The proposed method learns the nonlinear relationship between low resolution face image and high resolution face image in (nonlinear) kernel feature space. Super resolution imaging: A survey of current techniques [3] explains an insight into the stateof-the-art Super Resolution techniques and illustrated comparative experiments on real data that portrayed the robustness and utilization of all the methods.

\subsection{Super resolution based on MAP approach}

In this approach, the HR image is constructed via finding the HR image by maximizing conditional probability. The

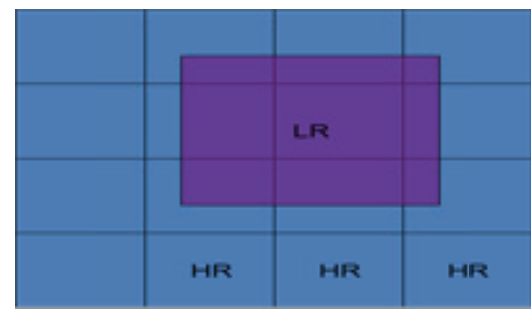

Fig. 3. SR based on MAP Approach

probability of $I_{h}$ provided $I_{1}$ has occurred

$$
\mathrm{P}\left(\mathrm{I}_{\mathrm{h}} \mid \mathrm{I}_{\mathrm{l}}\right)
$$

Generally $P\left(I_{1} \mid I_{h}\right)$ is modeled by the Gaussian distribution, so that maximizing $P\left(I_{l} \mid I_{h}\right)$ is equivalent to minimizing the data constraint [15] as follows: $I_{h}$ is the input high resolution image and the very low resolution image $\mathrm{I}_{1}$ is constructed by means of the Down sampling operator D. Each Low Resolution pixel is a linear combination of HR pixels as shown in Fig. 3.

Hence it is imperative that in order to minimize the probability, it is enough to minimize the data constraint

$$
\left\|\mathrm{DI}_{\mathrm{h}}-\mathrm{I}_{1}\right\|
$$

Different SR methods uses different techniques like to calculate the $\mathrm{I}_{\mathrm{h}}$.

\subsection{Example based approach}

In this method, the prior knowledge is used to reconstruct a HR image. It constructs the HR image by a linear combination of the HR training images.

Mathematically, the linear combination of LR images can be written as

$$
I_{h}^{\prime}=\sum w i \mathrm{I}_{\mathrm{h}}{ }^{\mathrm{i}}
$$

where $\mathrm{w}$ represents the weights and other $\mathrm{I}_{\mathrm{h}}{ }^{\mathrm{i}}$ represents the High resolution images from training pairs. We would like minimize the Euclidean distance between the reconstructed image and trained HR images. $\left\|\mathrm{I}_{1-} \sum w_{i} I_{l}^{i}\right\|$ is to be minimized. But we know that $I_{l}^{i}$ is obtained by down

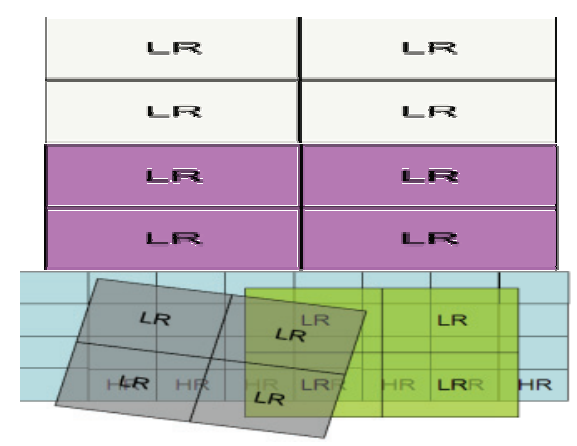

Fig. 4. Example based Approach 
sampling the input High resolution image shown in Fig. 4. Therefore, $\left\|\mathrm{I}_{1-} \sum w_{i} D H_{l}^{i}\right\|$ is to be minimized.

The greatest drawback of the method is that the large magnification factor causes overly smooth HR images which ultimately results in loss of fine details. Some of the popular example based methods are: Hallucination (Simon Baker and Takeo Kanade), Example Based, Single Image Super Resolution.

\section{Proposed Method}

An overview of the proposed method is shown in Fig. 5. The shortcomings those arise in the previous methods are mainly due to their inability to derive an association between the HR image space and the low resolution image space. Hence, this paper first attempts at deriving a relationship between HR and VLR image spaces. An association is learned in the training phase and is used to reconstruct the HR images in testing phase. The training phase consists of two steps, namely, modified centroid K means clustering and relationship learning. In the first step, a clustering algorithm is proposed as a pre-processing step.

\subsection{Association learning based super resolution}

Let the size of the image be $m \times n$. Then we will have exactly mn pixels.

The super resolution is the process of obtaining single image from multiple images of the same dimension by means of a linear combination of the given set of images, The super resolution image obtained from given set of high resolution image $\left\{h_{1}, h_{2}, h_{3}, \ldots h_{i}, \ldots h_{n}\right\}$ resolution images of the given person with different exposures is given by the following equation:

Let $h_{1}, h_{2}, h_{3}, \ldots . . h_{i}, \ldots h_{n}$ be the $n$ different high Let $a_{1}$, $a_{2}, a 3, \ldots \ldots a_{i}, \ldots a_{n}$ be the corresponding averages of luminance values of the given $n$ images. Then,

Total luminance value of the pixel $=$

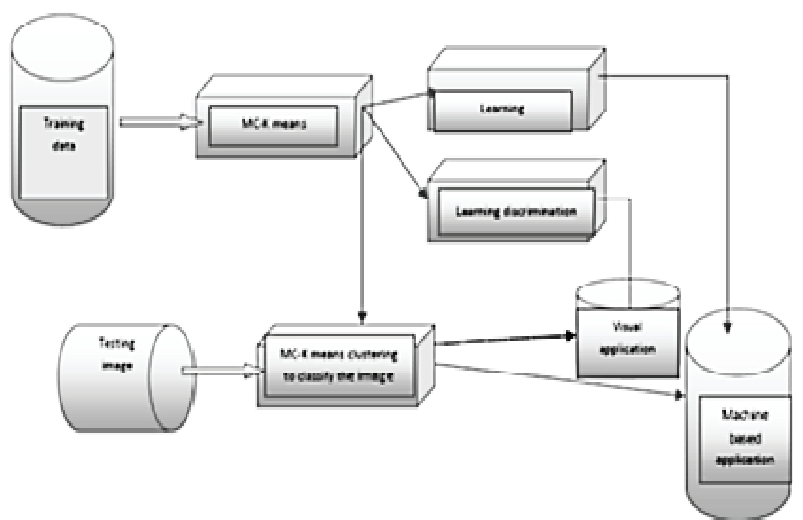

Fig. 5. Block diagram of the association based learning algorithm. $\sum_{i=0}^{n} \sum_{j=0}^{n} f(i, j)$
Average $\mathrm{a}_{\mathrm{k}}=\frac{\sum_{i=0}^{n} \sum_{j=0}^{n} f(i, j)}{m \times n}$ for $\mathrm{k}=1.2 \ldots \ldots . \mathrm{n}$

The average value of the pixel is calculated to calculate the appropriate value of the weigh to be given to each high resolution images $h_{i} I=1,2,3, \ldots . n$

Based on the average value, the weights $\mathrm{w}_{1}, \mathrm{w}_{2}$, $\mathrm{W}_{\mathrm{i}, \ldots .} \mathrm{W}_{\mathrm{n}}$ are calculated.

$$
\sum_{i=1}^{n} w_{\mathrm{I}} \mathrm{h}_{\mathrm{i}}=\mathrm{S}_{\mathrm{h}}
$$

It is well known facts that down sampling and stretch are linear operators.

Let $\mathrm{D}$ be the down sampling operator that acts on each set of high resolution images to produce corresponding set of very low resolution operator. The down sampled images are given by

$$
\begin{aligned}
& \text { Dh1 }=11, \mathrm{Dh} 2=12, \mathrm{Dh} 3 \\
& \text { Dhi }=1 \mathrm{i} \\
& \quad ; \\
& \quad ; \\
& \mathrm{Dhn}=\ln
\end{aligned}
$$

The super low resolution image obtained from given set of low resolution imags $\{11,12,13, \ldots \ldots .1 \mathrm{li}, \ldots \ln \}$ is given by

$$
\sum_{i=1}^{n} w_{\mathrm{I}} \mathrm{l}_{\mathrm{i}}=\mathrm{L}_{\mathrm{h}}
$$

The same weight is used here to obtain the SR image for the low resolution image also.

From (5)

We $\sum_{i=1}^{n} w_{\mathrm{I}} 1_{\mathrm{i}}=\mathrm{S}_{1}$

$$
\sum_{i=1}^{n} w_{\mathrm{I}} \mathrm{Dh}_{\mathrm{i}}=\mathrm{L}_{\mathrm{h}} \text { by (4) }
$$

Since D is a linear operator, we can rewrite (4) as

$$
\begin{aligned}
& \mathrm{D}\left(\sum_{i=1}^{n} w_{\mathrm{I}} \mathrm{h}_{\mathrm{i}}\right)=\mathrm{S}_{1} \\
& \mathrm{D} \mathrm{S}_{\mathrm{h}}=\mathrm{S}_{1}
\end{aligned}
$$

Since $\mathrm{Dhn}=\ln$, the high resolution can be obtained by stretching the corresponding low resolution image.

$$
\text { hi }=\mathrm{D} \ln
$$

From (6) we can understand that Super resolution of VLR images can be obtained from the SR image of all high resolution images by just down sampling it.

Let $\mathrm{dhi}=\mathrm{Shi}-\mathrm{Hi}, \mathrm{I}=1, .2, \ldots \ldots \ldots \mathrm{n}$ be the difference image between the super resolution image and HR image hi.

Then,

$$
\sum_{i=1}^{n} \cdot \mathrm{dh}_{\mathrm{i}}=\mathrm{nd}_{\mathrm{h}}
$$


For a single individual, we would be having a set of images, which would be conpriseing of all its low and high resolution images. We consider this set as one cluster and ( $\mathrm{Si}, \mathrm{Sh}$ ) would be its center.

Let $\mathrm{R}$ denotes the association relationship map from VLR - HLR training images.

For each cluster, we denote the training image pairs by $T=\left\{I_{l}^{i}, I_{h}^{i} \mid i=1, \ldots, N\right\}$

$$
I_{h}^{i}=R\left(I_{l}^{i}\right)
$$

For each new input VLR - HLR mage for that group, the new found association map is applied to the VLR image and the corresponding high super resolution images is found and is compared with the available HLR image and mean square error method is used to reduce the reconstruction error.

$$
\tilde{I}_{h}=R I_{l} \text { and } I_{h}=\tilde{I}_{h}+\varepsilon
$$

The reconstruction error is calculated,

$$
e_{R}\left(I_{h}\right)=\left\|I_{h}-\tilde{I}_{h}\right\|^{2}
$$

And it is to be made sure that it is minimum and which would help us learn the relationship

$$
\begin{aligned}
R & =\arg \min _{R} \mathbf{E}\left(e_{R}\left(I_{h}\right)\right) \\
& =\arg \min _{R} \int e_{R}\left(I_{h}\right) p\left(I_{h}\right) d I_{h} \\
& \approx \arg \min _{R} \sum_{i} \frac{1}{N} e_{R}\left(I_{h}^{i}\right) \\
& =\arg \min _{R} \frac{1}{N} \sum_{i}\left\|I_{h}^{i}-R I_{l}^{i}\right\|
\end{aligned}
$$

\subsection{Linearity clustering}

Improved $\mathrm{k}$ clustering is employed here, by which the linear relationship, that is expected to be there between the input and corresponding HLR is ensured in the clustered training image pairs

\subsubsection{Modified centroid $k$ means clustering algorithm}

A cluster is a collection of pixels which are similar between themselves. Clustering the image is the process of grouping the pixels according to some characteristics such as pixel intensity, distance criterion etc. Clustering is the most important unsupervised learning problem. Here the algorithm classifies object automatically based on the criteria given by the user K-means clustering algorithm is an exclusive clustering algorithm in the sense that if a certain pixel belongs to a definite cluster then it could not be included in another cluster.

In the K-means clustering algorithm, initially we have to define the number of clusters $\mathrm{k}$. Initial cluster centers have to be found. In the standard K- means clustering algorithm, initial cluster centers are found in random but here, in our work, a new method for defining initial cluster centers is proposed. Difference between the maximum and minimum pixel of an input image is divided by the number of clusters $\mathrm{k}$ which gives an interval. Initial centers for the clusters are chosen using this interval such that each cluster center varies with a fixed range among themselves. After finding initial cluster centers the distance between each input image pixel to each cluster centers are calculated. The distance is calculated by finding the absolute difference between a pixel and a cluster center. The pixel is moved to particular cluster which has shortest distance among all. Then the centroid is re-estimated. It is done by finding the average of the pixels in the clusters got. Again each pixel is compared against each centroid. The process continuous until the center converges. The main aim is to minimize an objective function.

\section{Modified Centroid K Means Clustering Algorithm Steps:}

1. Let $\mathrm{y} 1, \ldots, \mathrm{yM}$ are $\mathrm{L}$ data points in the input image or input dataset, $\mathrm{k}$ be the number of clusters, and $\mathrm{w} 1, \ldots$, wK be k cluster centers.

2. Find the maximum and minimum pixel or data point from the input image or input dataset.

3. Their difference is found and it is divided by $\mathrm{K}$ clusters which gives an interval.

4. In a loop determined by number of clusters using the interval got from step 3, find the initial centroids of the $\mathrm{k}$ clusters such that each cluster center (centroid) varies with a fixed range amongst them.

5. Distance between each pixel and each cluster center is found.

6. The distance function is given by $J=\left|x_{i}-c_{j}\right|$ For $i=1, \ldots, N$ and for $j=1, \ldots, k$ Where $\left|x_{i}-c_{j}\right|$, the absolute difference of the distance between a data point $x_{i}$ and the cluster center $c_{j}$ is an indicator of the distance of the $\mathrm{N}$ data points from their respective cluster centers.

7. Distribute the data points $\mathrm{x}$ among the $\mathrm{k}$ clusters using the relation $y \in C_{j}$ if $\left|x-c_{j}\right|<\left|x-c_{i}\right|$ for $i=1,2, \ldots, k, i \neq j$, where $C_{j}$ denotes the set of data points whose cluster center is $\mathrm{c}_{\mathrm{j}}$.

8. Updated cluster center is given as, $\mathrm{c}_{\mathrm{i}}=\frac{1}{\mathrm{~m}_{\mathrm{i}}} \sum_{\mathrm{x} \in \mathrm{C}_{\mathrm{i}}} \mathrm{x}$, for $\mathrm{i}=1, \ldots, \mathrm{k}$, where $\mathrm{m}_{\mathrm{i}}$ is the number of objects in the dataset $C_{i}$, where $C_{i}$ is the $i^{\text {th }}$ cluster and $c_{i}$ is the centre of cluster $\mathrm{C}_{\mathrm{i}}$.

9. Repeat from Step 5 to Step 8 till convergence is met (old and new centroids remain same).

\section{Algorithm}

Association Learning relationship mapping $R$ from training data

Require: training image pairs $\left\{I_{l}^{i}, I_{h}^{i}\right\}$ terminating condition $\in$, initial relationship $R^{(0)}$, step $\alpha_{0}$ 
1: $n \leftarrow 1$

2: $d^{(0)} \leftarrow\left(\partial \mathcal{E}\left(R^{(0)}\right) / \partial R\right)^{T}$

3: while $\left\|d^{(n-1)}\right\|>\in$ do

4: find the optimal $\tilde{\alpha} \in\left[\begin{array}{ll}0 & \alpha_{0}\end{array}\right]$ that minimizes $f(\alpha)=$ $\mathcal{E}\left(R^{(n-1)}-\alpha d^{(n-1)}\right)$

5: update $: R^{(n)} \leftarrow R^{(n-1)}-\tilde{\alpha} d^{(n-1)}$

6: $n \leftarrow n+1$

7: end while

8: return $R^{(n)}$ as the learned relationship mapping

\section{Experiments}

\subsection{Performance analysis of VLR algorithm}

Face Recognition appears to be relatively a trivial task; however, the task contains a lot of challenges. In this paper, the algorithm developed for VLR problems are designed to address those challenges and come out with successful results. Since Extracting image from VLR and its success largely depends on learning process.

For human based visual detection and machine based face detection, this paper has suggested two different methods and the experiments are conducted to evaluate the performance of the algorithms. The following popular methods that have been used in the literature are taken to compare the performance of the proposed methods.

1. Hallucinating face (HLF),

2. Eigen transformation-based face SR (ETF),

3. Kernel prior face SR (KPF),

4. Position face hallucination (PFH),

5. Relation based face recognition [RLSR]

The study of the outcomes of the proposed method confirms that it surpasses the existing methods in terms of visual quality and recognition performance.

\subsubsection{Experiment for human based face detection}

Super resolution is the process of constructing the high resolution image from many very low resolution images. As explained before, the association relation that could exist between the high resolution image space and very low resolution image space is used to construct the HR image from the very low resolution query image (obtained from videos of CCTV etc). Then, quality assessment of the reconstructed HR image is done. Normally, the visual quality assessment is categorized into two: first assessment is based on human and is called subjective visual quality; second one is objective visual quality, which is based on any statistical measure. Here, in this paper both methods are employed to assess the visual quality of the reconstructed image; first method employs the opinion of the humans and second one uses mean squared error.

Database and Settings: Public face databases taken and our own created database containing images of hundred people with different expressions and exposures are used for the experiments. From the database, a subset that consists of 500 frontal view face images (one image per individual) is used. From Indian database, a subset of frontal view images of 38 persons with 64 different illuminations is used. All images are manually aligned by the position of the eyes and normalized to the resolutions of $64 \times 48(\mathrm{HR})$ and $8 \times 8(\mathrm{VLR})$.

Experiments are conducted on 2 databases, one public database and another locally created. The images in the two databases are divided into two disjoint sets. Division is carried out based on their class labels. The 200 images from Indian databases are randomly selected for training and classifying and the rest of the data are used for testing purpose. Similarly a data size of 50 images from our own database is kept for training and the remaining 50 images are used for testing purposes.

The high resolution images and the corresponding low resolution images are clustered using $\mathrm{k}$ means algorithms. For each input image with different exposures and different resolutions, the corresponding LVR images are created and the low resolution images are super resoluted to form a HR image and in the process, an association is learned. Thus each cluster will represent a particular kind of association and that association is very much used later to identity where the input low resolution images are to be associated in the testing phase

Thus, the VLR testing image will be classified into one of the clusters, and then, the reconstructed image is obtained by applying associated learning. Ma, J. Zhang, and C. Qi, suggested one way of improving the quality of the image in which each image is divided into small patches, and super resolution is done patch by patch and is overlapped with its neighboring pictures to avoid the blocky effects.

Results: The very low resolution images from our own database and Indian database are taken and the proposed algorithm is applied on these images and the performance of the algorithm is compared with other prominent algorithms in the literature and the results are shown in the Figs. 6, 7. Fig. 6 shows very low resolution input images of several persons taken from our database and their corresponding output images by different methods and the proposed method. Fig. 6(a) and 6(h) show the input very low resolution query image and its original HR image, respectively. Fig. 6(b)-(f) displays the results using the various methods (b) PFH. (c) HLF. (d) ETF. (e) KPF. (f) RLSR. (g) Proposed ALSR.

Most of the existing face recognition methods are incapable to unfailingly identify persons or worse it will recognize the incorrect person. Face Images of same person with different expressions, postures, and exposures have to be promptly recognized. It is highly ideal to get the images which are center, portrait type environment for the system to identify the faces correctly. Getting such images are highly improbable. This paper has introduced methods 

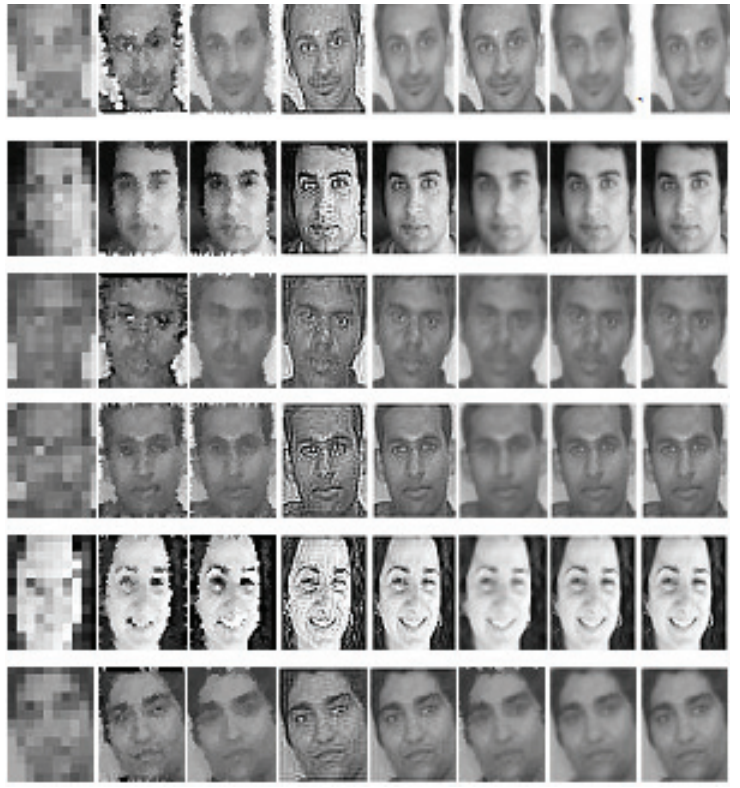
(a)
(b)
(c)
(d)
(e)
(f) $\quad(\mathrm{g})$
(h)

Fig. 6. Super Resolution results on the OUR-OWN database. Very low resolution Images of different persons: (a) Input VLR image; (b) PFH; (c) HLF [1] ; (d) ETF; (e) KPF [2] ; (f) RLSR; (g) Proposed ALSR; (h) Original HR image.

that overcome the above said difficulties and experimental results shown in Figs. 6, 7 greatly corroborate our claim.

Fig. 6 shows how our algorithm produces better results for those very low resolution images of sizes as low as $8 \times 8$ of different persons and reconstructs the corresponding high resolution images. Reconstructing the face images is quite challenging and is one factor that highly affects recognition performance of any algorithm. The Fig. 6(a) shows the original VLR images. Figs. 6(b) to 6(f) represent the reconstruction results of the existing methods (HLF) [1] (ETF), (KPF) [2], (PFH) [18], [RLSR] respectively which are taken for comparison. Fig. 6(g) shows the reconstructed HR image from the corresponding VLR image.

Fig. 7 shows how our algorithm produces better results for those very low resolution images of sizes as low as $8 \times 8$ of with different expressions and reconstructs the corresponding high resolution images.

From the Figs. 6, 7, it is well understood that for a very low resolution image of size $8 \times 8$, KPF method gives relatively blurred image. With the size of VLR images with low dimensions as $8 \times 8$ the algorithm fails to produce many high frequency details. The visual quality of the reconstructed image by ETF method is reasonably good. But, high resolution images produced by this algorithms lack the look and feel of the original High resolution images as the some important high frequency details are lost. The reconstructed images by the HLF method are better when compared with KPF method but it still has some blurring effect. The reconstructed images produced by ALSR method are better in quality and appearance

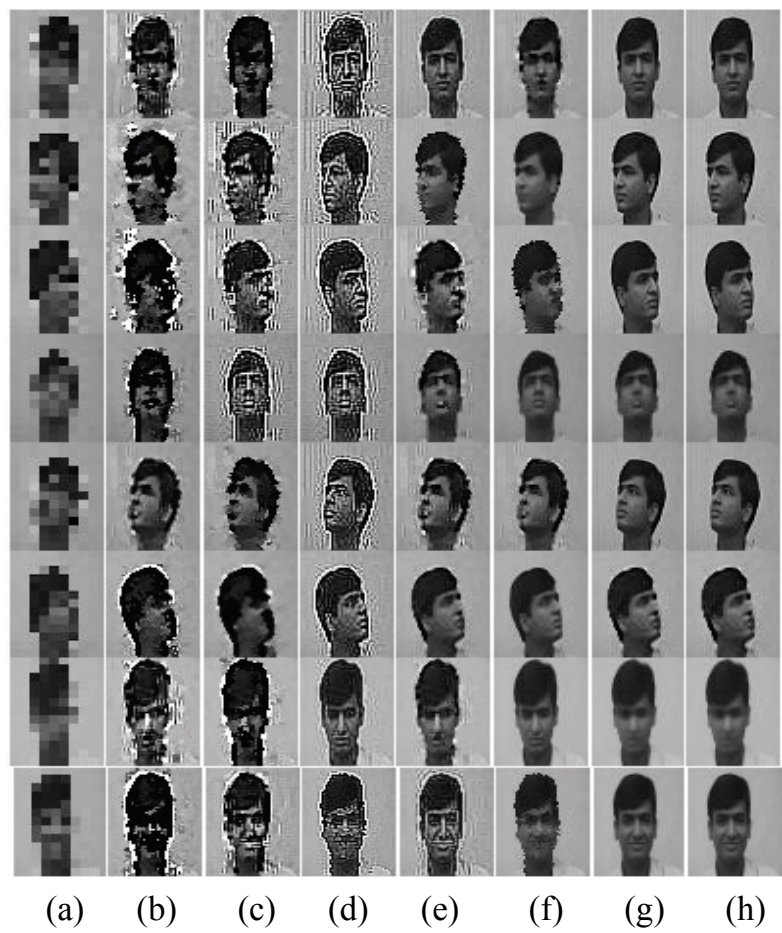

Fig. 7. Super Resolution results on the Indian database with different positions: (a) Input VLR image; (b) PFH; (c) HLF [1]; (d) ETF; (e) KPF [2]; (f) RLSR; (g) Proposed ALSR; (h) Original HR image.

when compared with all other four methods but it lacks clarity when compared with the original image. The method proposed in this paper can easily recover the images with face variations and lighting variations. When compared other existing methods which are taken for comparison, this algorithm produces better results and reconstructed images are close to the original HR images. Moreover, the times taken by PFH and RLSR algorithms to reconstruct the original images are $43 \mathrm{~s}$ and $30 \mathrm{~s}$ respectively. But, our proposed method is implemented in Matlab and FPGA, and it takes $24 \mathrm{~s}$ when implemented in matlab and $18 \mathrm{~s}$ when implemented in FPGA.

Moreover, Table 1 shows the Root Mean Square Error (RMSE) values of different super resolution methods for our own database and Indian database. The recorded values clearly show that our algorithm has low RMSE and hence it performs better when compared to every other SR methods under consideration. Fig. 8 shows the real time implemention of face recognition in FPGA.

Table 1. Root Mean Square Error(RMSE)

\begin{tabular}{c|c|c|c|c|c|c}
\hline \multicolumn{7}{c}{ RMSE Of Different SR Methods for Database } \\
\hline Data base & PFH & HLF & ETF & KPF & RLSR & ALSR \\
\hline Indian data base & 1090.2 & 1056.5 & 1002.7 & 1101.8 & 770.7 & 670.4 \\
\hline Our data base & 987.8 & 992.7 & 972.6 & 943.8 & 745.9 & 657.3 \\
\hline
\end{tabular}

\subsection{Experiment 2: Recognition performance}

This part of this paper describes the superiority of the 

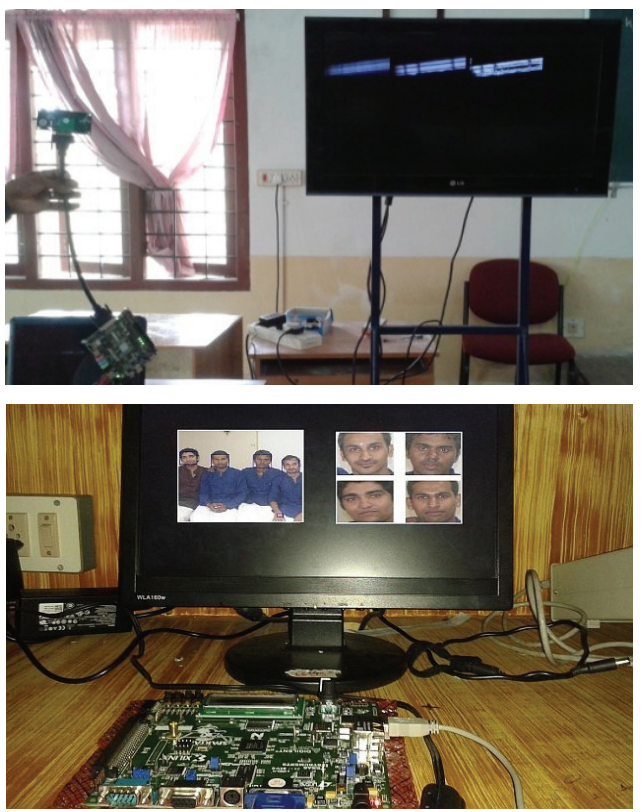

Fig. 8. Real time setup for face recognition in FPGA

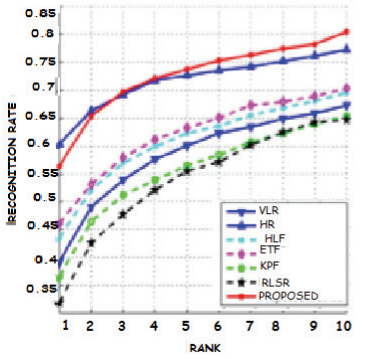

(a)

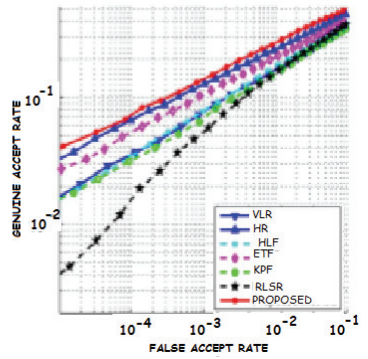

(b)
Fig. 9. Recognition results on the created Database with PCA and INN face recognition Engine: (a) CMC; (b) $\mathrm{ROC}$

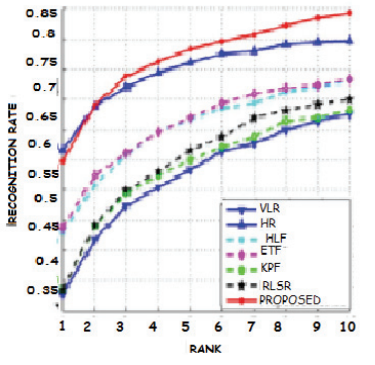

(a)

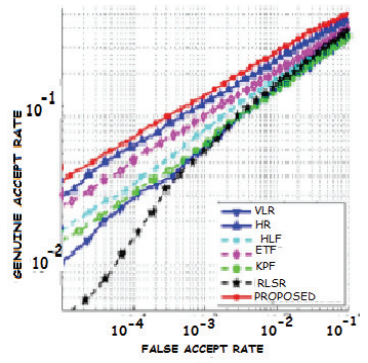

(b)
Fig. 10. Recognition results on the Indian Database with PCA and INN face recognition Engine: (a) CMC; (b) ROC

proposed algorithm in terms of its performance. The performance is measured by measuring the recognition accuracy. The recognition accuracy is evaluated by means of false acceptance rate (FAR). The FAR means that at what rate the system accepts incorrect input as a positive match. For every ten thousand inputs, an error of I false recognition is accepted. Due care need to be taken to ensure that our system has very low false rejection rate also. For this part of the experiment, the images from our own database, Indian database, GTAV and LFW (labeled Faces in the wild) database were taken. The reason why various databases used in this experiment are: variations in face positions, variations in expressions, variations in illuminations, variation in clothing, variations in ethnicity, and variations in background. The experimental results are organized as follows: Part A reports the performance of Distinguishing-Super-Resolution method on our own database and Indian database using face recognition algorithms, namely, PCA and eigen faces algorithm, a combination of PCA and LDA algorithm, face recognition based on SVM machines is shown in Fig. 9; Part B investigates the performance of Distinguishing-SuperResolution-method on query images with different (low) resolutions is shown in Fig. 10; Part $\mathrm{C}$ further evaluates the proposed method on Extended Yale Face database, CMUPIE and surveillance camera face (SCface) databases in Figs. 11, 12.

Databases and Settings: The databases used for this experiment are our developed database, Indian data base, CAS-PEAL face database, Extended Yale Face database. Extended Yale Face Database B is mainly used for images with different poses and different illumination conditions. It contains 16128 images. For CAS-PEAL database, 20 images with a frontal view, 20 images with different pose angles, 20 images with different facial expressions like

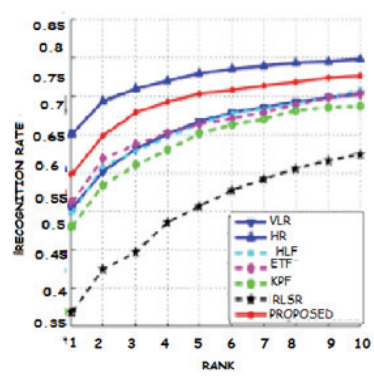

(a)

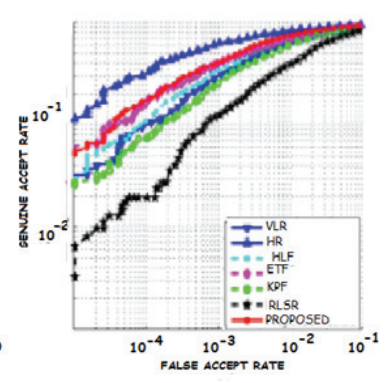

(b)
Fig. 11. Recognition results on the created Database with PCA and SVM face recognition Engine: (a) CMC; (b) ROC

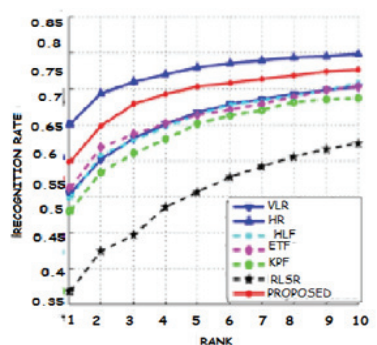

(a)

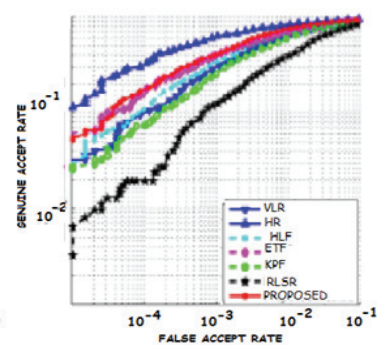

(b)
Fig. 12. Recognition results with the Kernel L DA + INN face recognition engine: (a) $\mathrm{CMC}$; (b) ROC 
neutral, eye closing, frown, smile, surprise, and mouth open are used. The database contains nearly 1040 face images and a subset of the database is used for this experiment. The same is repeated in the case of other databases also. Only the frontal view images from the CMU-PIE database are used. A part of the image set is used as the training set and another portion of the image set is used in the training phase. The test results are very encouraging for those frontal images and for those images with face variations, differing poses, smiles, etc,

Results and Analysis: Five existing algorithms have been taken to compare the performance of the proposed method. The experiment is carried out using the reconstructed HR images evaluate the performance of the proposed method on a face recognition system; we carry out the experiments using the reconstructed HR images with existing face recognition engines. The VLR and original HR images are used as benchmark performance, and five existing SR algorithms namely PFH, ETF, KPF, HLF and RLSR are used for comparison.

Part A: describes the performance of our discriminative method on the Indian database: In this experiment In Fig. 9-18, face recognition algorithms have been employed to evaluate the performance of the algorithm.

$$
\begin{aligned}
& \text { PCA }+1 \mathrm{NN}, \\
& \text { PCA SVM, } \\
& \text { kernel PCA }+1 \mathrm{NN}, \\
& \text { kernel LDA }+1 \mathrm{NN}
\end{aligned}
$$

In $\mathrm{PCA}+1 \mathrm{NN}$ face recognition algorithm, PCA is used to extract the eigenfaces and will be used acurve which helps analyze the performance of the system and CMC-

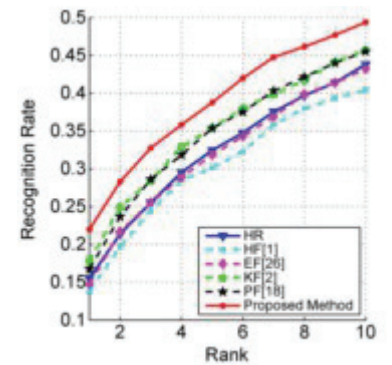

(a)

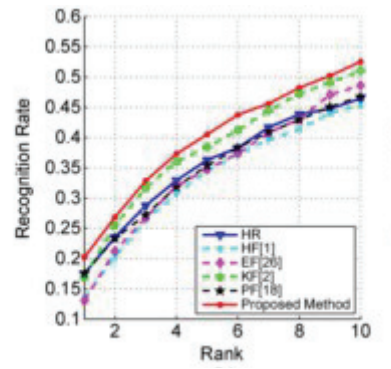

(b)

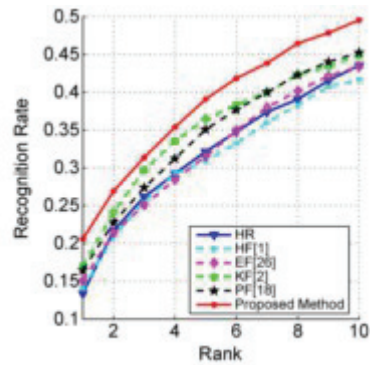

(c)

Fig. 13. Results (ROC curves) of the proposed method on images with different resolutions. The resolution of the probe image for LR is (a) 76, (b) 14 12, and (c) 2824.

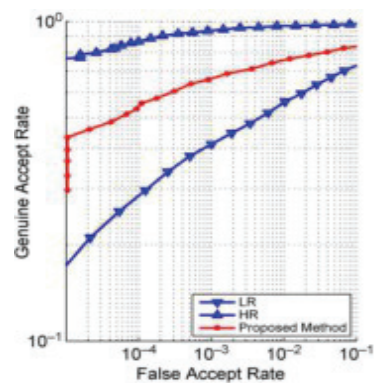

(a)

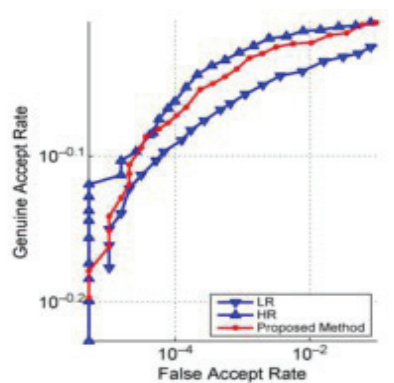

(b)

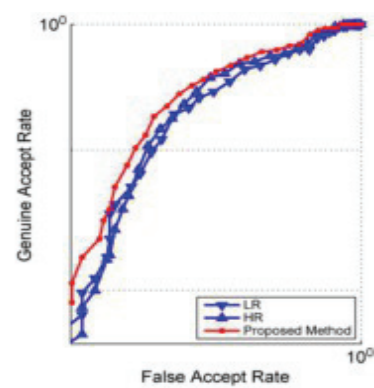

(c)

Fig. 14. Recognition results (CMC curves) on the CMU-PIE database using different face recognition algorithms: (a) PCA 1NN; (b) Kernel PCA1NN; (c) PCASVM

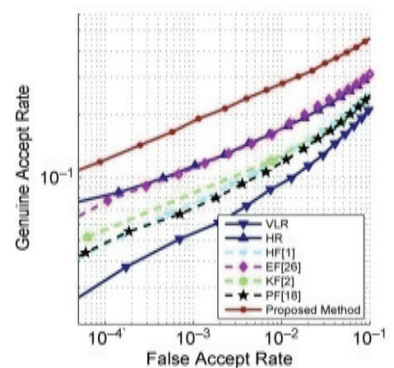

(a)

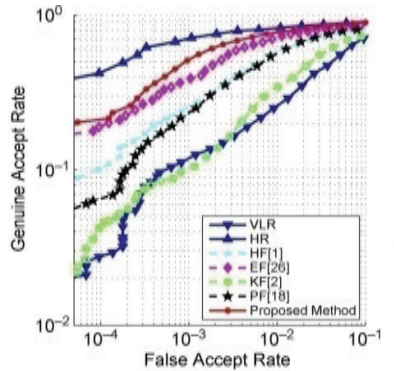

(b)

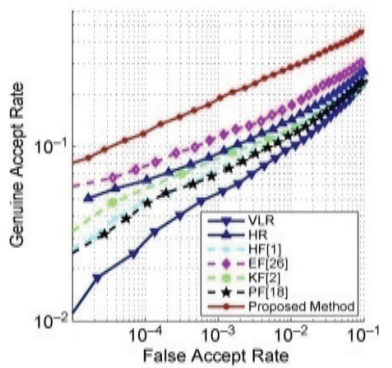

(c)

Fig. 15. Recognition results (CMC curves) on the SCface database using different face recognition alg: (a) PCA 1NN; (b) Kernel PCA1NN; (c)PCASVM 


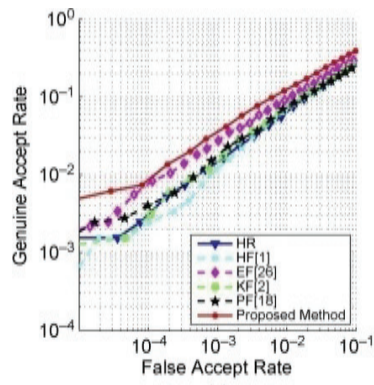

(a)

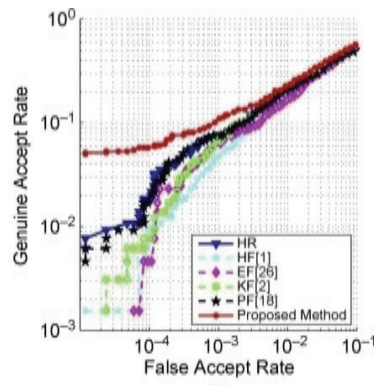

(b)

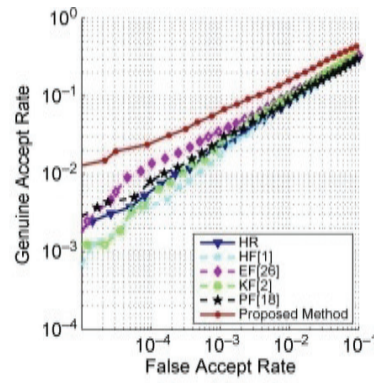

(c)

Fig. 16. Recognition results (ROC curves) on the CMU-PIE db using different algo: (a) PCA1NN; (b) Kernel PCA1NN; (c) PCA SVM.

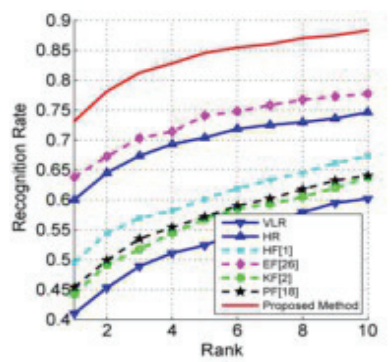

(a)

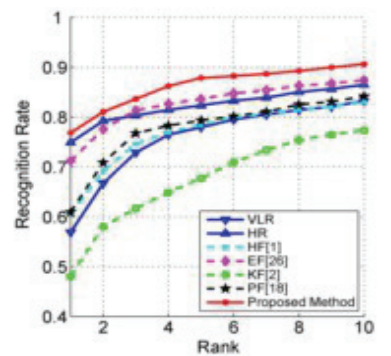

(b)

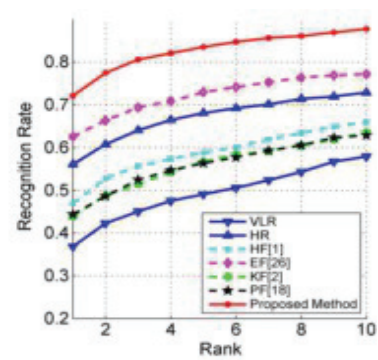

(c)

Fig. 17. Recognition results (ROC curves) on the CMU-PIE database using different face recognition algorithms: (a) PCA1NN; (b) Kernel PCA-1NN; (c) PCA SVM

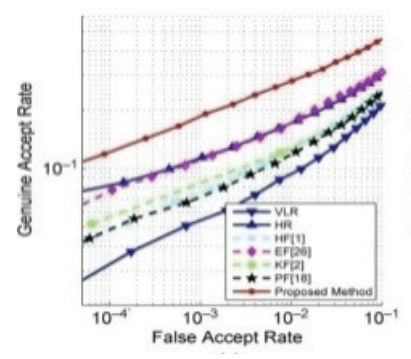

(a)

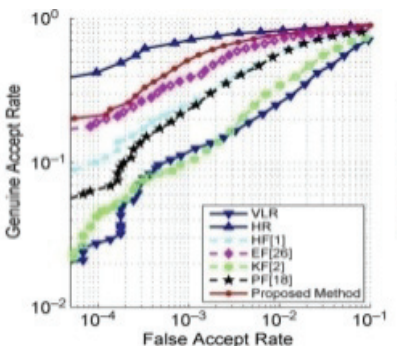

(b)

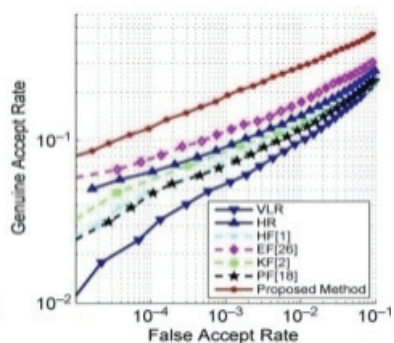

(c)

Fig. 18. Recognition results (ROC curves) on the SCface database using different face recognition algorithms: (a) PCA1NN; (b) Kernel PCA1NN; (c) PCA-SVM.

Cumulative match Characteristic as the feature for the $1 \mathrm{NN}$ classifier. $95 \%$ of the variance is used in the training phase of PCA. Fig 10a clearly reveals that recognition accuracy has come down markedly with VLR image when compared to the HR image. Marginal improvement is noticed when HLF and ETF algorithms are employed. RLSR performs better. The performance has dropped when other two existing algorithms are employed. Our method improves the performance and is evident from the figure and has improved it to a tune of $16 \%$. Fig. 11 shows the performance when kernel PCA is used as the feature extractor. The other things remain the same and no noticeable difference is found. Further experiments are conducted with distinguishable face recognition algorithms and kernel LDA 1NN [14] and PCA SVM, as the face recognition algorithms. Kernel functions for Kernel LDA and State vector machine are Gaussian radial basis function and Inner product respectively. The results are shown in Fig. 12 and Fig. 13. The performance has seen a drop of $20 \%$ for VLR method has shown $6 \%$.

A receiver operating characteristic (ROC) curve is curve is a curve which highly points the increase in the identification rate of the system. ROC Curve and the CMC are drawn and they depict how our method outperforms other method.

\section{Conclusion}

He very low resolution face recognition problem has been one of the most important face recognition problem and solution to such a problem has assumed much significance, for, in the changing requirements, the application based on this are wide and varied. Hence, this paper has defined and discussed the very low resolution problem, and proposed one good algorithm which used 
ordinary least square method of linear regression model and a novel association relationship based SR algorithm. Based on this idea, for good visual quality applications, a new data constraint that measures the error in the HR image space was developed, and RLSR was proposed. For machine-based face recognition applications, a discriminative constraint was designed and integrated with the new data constraint, and DSR was proposed. Experimental results show that the proposed method outperforms the existing SR algorithms in terms of visual quality and recognition performance.

\section{References}

[1] S. Baker and T. Kanade, "Limits on super-resolution and how to break them," IEEE Trans. Pattern Anal. Mach. Intell., vol. 24, no. 9, pp.1167-1183, Sep. 2002.

[2] A. Chakrabarti, A. N. Rajagopalan, and R. Chellappa, "Super-resolu tion of face images using kernel PCAbased prior," IEEE Trans. Multimedia, vol. 9, no. 4, pp. 888-892, Jun. 2007.

[3] G. Cristóbal, E. Gil, F. S̆ ${ }_{\text {roubek}}$, J. Flusser, C. Miravet, and F. Rodria-cute; guez, uperresolution imaging: A survey of current techniques," in Proc. Adv. Signal Process. Algorithms, rchitectures, Implementations XVIII, 2008, vol. 7074, pp. 0C1-0C18.

[4] W. Freeman, E. Pasztor, and O. Carmichael, "Learning low-level vision,” Int. J. Comput. Vis., vol. 40, no. 1, pp. 25-47, Oct. 2000.

[5] W. Gao, B. Cao, S. Shan, X. Chen, D. Zhou, X. Zhang, and D. Zhao, "The CAS-PEAL large- scale Chinese face database and baseline evaluations," IEEE Trans. Syst., Man, Cybern A, Syst., Humans, vol. 38, no. 1, pp. 149-161, Jan. 2008.

[6] A. Georghiades, P. Belhumeur, and D. Kriegman, "From few to many: Illumination cone models for face recognition under variable lighting and pose," IEEE Trans. Pattern Anal. Mach. Intell., vol. 23, no. 6, pp. 643-660, Jun. 2001.

[7] D. Glasner, S. Bagon, and M. Irani, "Super-resolution from a single image," in Proc. IEEE Int. Conf. Comput. Vis., 2009, pp. 349-356.

[8] M. Grgic, K. Delac, and S. Grgic, "SCface-surveillance cameras face database," Multimedia Tools Appl. J., vol. 51, no. 3, pp. 863-879, Feb. 2011.

[9] P. H. Hennings-Yeomans, S. Baker, and B. Kumar, "Simultaneous super-resolution and feature extraction for recognition of low-resolution faces," in Proc. IEEE Int. Conf. Comput. Vis. Pattern Recognit., 2008, pp.1-8.

[10] K. Jia and S. Gong, "Generalized face super-resolution,” IEEE Trans. Image Process., vol. 17, no. 6, pp. 873-886, Jun. 2008.

[11] K. Kim and Y. Kwon, "Single-image super-resolution using sparse regression and natural image prior," IEEE Trans. Pattern Anal. Mach. Intell., vol. 32, no. 6, pp. 1127-1133, Jun. 2010.

[12] B. Li, H. Chang, S. Shan, X. Chen, and W. Gao, "Hallucinating facial images and features," in Proc. IEEE Int. Conf. Pattern Recognit., 2008,

13] H. Li, T. Jiang, and K. Zhang, "Efficient and robust feature extraction by maximum margin criterion," IEEE Trans. Neural Netw., vol. 17, no.1, pp. 157-165, Jan.2006.

[14] C. Liu, "Capitalize on dimensionality increasing techniques for improving face recognition grand challenge performance,” IEEE TransPattern Anal. Mach. Intell., vol. 28, no.5, pp. 725-737, May 2006.

[15] C. Liu, H. Y. Shum, and W. T. Freeman, "Face hallucination: Theoryand practice," Int. J. Comput. Vis., vol. 75, no. 1, pp. 115-134, Oct. 2007.

[16] W. Liu, D. Lin, and X. Tang, "Hallucinating faces: Tensorpatch super-resolution and coupled residue compensation," in Proc. IEEE Int. Conf.Comput. Vision Pattern Recognit., 2005, pp. 478-484.

[17] Y. M. Lui, D. Bolme, B. A. Draper, J. R. Beveridge, G. Givens, and P.J. Phillips, "A meta-analysis of face recognition covariates," in Proc.Int. Conf. Biometrics: Theory, Appl. Syst., 2009, pp. 1-8.

[18] X. Ma, J. Zhang, and C. Qi, "Hallucinating face by position-patch," Pattern Recognit., vol. 43, no. 6, pp. 2224-2236, Jun. 2010.

[19] J. S. Park and S. W. Lee, "An example-based face hallucination method for single-frame, low-resolution facial images," IEEE Trans. Image Process., vol. 17, no. 10, pp. 1806-1816, Oct. 2008.

[20] W. W. Zou and P. C. Yuen, "Face superresolution," in Emerging Topics in Computer Vision and its Applications, C. Chen, Ed. Singapore: World Scientific, 2011, ch. 2 .

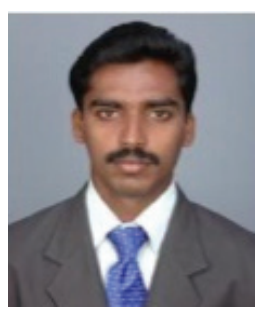

C. Senthilsingh received the B.E. degree in Electrical and Electronics Engineering from the MS University, India, M-Tech VLSI Design. His research interest includes VLSI, Image processing wireless communication, Neural Networks and fuzzy logic, Communication networks

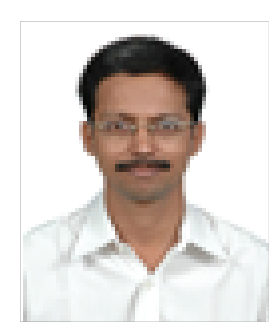

M. Manikandan received the B.E. and M.E degree from madras University, Chennai, India, in 2000. He completed his $\mathrm{PhD}$ in Anna University and currently working in electronics engineering department in Anna University, chennai, India. His research interest includes Image Processing, VLSI, Sensor Networks and signal processors. 